\title{
Simulação Numérica do Crescimento da Lentilha de Solda Obtida pelo Processo de Soldagem a Ponto por Resistência Elétrica no Aço Inox AISI 304
}

(Numerical Simulation of Nugget Growth for Resistance Spot Welding in Stainless Steel AISI 304)

\author{
Carlos Henrique de Andrade Molenda", , Aleir Antônio Fontana de Paris ${ }^{1}$, Inácio da Fontoura Limberger ${ }^{1}$, Rogério Brittes da Silva ${ }^{2}$, \\ Leandro Marin de Oliveira ${ }^{3}$, Luiz Antônio Righi', \\ IUniversidade Federal de Santa Maria, Departamento de Engenharia Mecânica, Santa Maria, RS, Brasil, \\ molenda@smail.ufsm.br,aparis@ct.ufsm.br \\ 2Universidade Federal do Rio Grande do Sul, PROMEC, \\ 3WEG Equipamentos Elétricos S., Jaraguá do Sul, SC, Brasil, \\ 4Universidade Federal de Santa Maria, Departamento de Eletromecânica e Sistemas de Potência, Santa Maria, RS, Brasil, \\ righi.luiz@gmail.com
}

\section{Resumo}

O crescimento das lentilhas de solda obtidas pelo processo de soldagem a ponto por resistência elétrica em chapas de aço inoxidável AISI 304 foi analisado através de simulação numérica com o método de elementos finitos, sendo utilizado para essa finalidade o programa comercial ANSYS. O modelo matemático utilizado considerou: acoplamento entre os campos elétricos e térmicos em regime transiente; a variação das propriedades elétricas e térmicas do aço AISI 304 com a temperatura e condições de contorno térmicas de convecção. A geometria tridimensional analisada, axissimétrica, permitiu a simplificação para uma geometria bidimensional. Os resultados obtidos com o modelo foram comparados com resultados experimentais apresentados na literatura. Foram consideradas espessuras de chapas de 1, 2 e $3 \mathrm{~mm}$, e diferentes diâmetros de face para os eletrodos. As simulações determinaram os tempos necessários de aplicação de diferentes valores de corrente para obtenção dos diâmetros e penetração das lentilhas de solda recomendados em cada situação. Os resultados mostraram também que com o uso de maiores correntes menos energia é consumida na formação da lentilha de solda.

Palavras-chave: Solda a ponto; Simulação numérica; Aço inoxidável.

Abstract: The growth of the spot welding nugget in AISI 304 stainless steel sheets was analyzed by numerical simulation with the finite element method, being used for this purpose the commercial code ANSYS. The mathematical model considers: coupling between the electric and thermal fields in transient regimen; temperature dependent thermal and electrical properties of AISI 304 steel; convection thermal boundary condition. The analyzed three-dimensional geometry, axisymmetric, allowed the simplification to a two-dimensional geometry. The results of the model were compared with experimental results presented in the literature. Sheets thickness of 1, 2 and 3 $\mathrm{mm}$, and different diameters of electrodes faces were considered. The results allowed determining the necessary current application times for attainment the recommended diameter and penetration of the nugget in each situation. The results also showed that with the use of larger currents the nugget formation consumes less energy.

Keywords: Spot Welding; Numerical Simulation; Stainless Steel

\section{Introdução}

O processo de soldagem a ponto por resistência elétrica, RSW (Resistance Spot Welding), é entre os processos de soldagem por resistência elétrica, o mais utilizado. Por ser adequado para união de chapas metálicas finas, tem grande emprego em diversas indústrias, notadamente na indústria automobilística onde possibilita grande produtividade, custo relativamente baixo e atende os requisitos de qualidade. Largamente utilizada para soldagem de aço carbono comum, sua aplicação na soldagem

(Recebido em 20/11/2009; Texto final em 25/11/2010). de outros materiais tem gerado o desenvolvimento de pesquisas para melhoria dos processos [1-3]. Num mercado extremamente competitivo, a procura por melhor qualidade e redução de custos é imperativa. No que se refere ao RSW isso exige um melhor conhecimento do mecanismo de formação da lentilha de solda e determinação da influência dos diversos parâmetros do processo de soldagem na sua geometria.

No RSW interagem fenômenos mecânicos, elétricos, térmicos e metalúrgicos, em um intervalo de tempo muito pequeno. Por isso, sua análise experimental é complexa, exigindo grande dispêndio de recursos na tarefa de determinação da função dos diversos parâmetros que influenciam na qualidade do resultado, o ponto de solda. Uma alternativa que tem sido utilizada é a abordagem teórica com o uso do método de elementos finitos para resolução das equações resultantes do modelo matemático [4-7]. A análise por simulação numérica tem a grande vantagem 
da economia de tempo e recursos, permitindo facilmente a variação dos diferentes parâmetros que afetam o processo de soldagem. Além disso, facilita a verificação da importância de determinado parâmetro através da simplificação dos modelos. Desconsiderando-se alguns aspectos complicadores no modelo matemático, os efeitos dos fenômenos remanescentes no modelo podem ser melhor avaliados. No mercado encontram-se alguns programas baseados no método de elementos finitos que podem ser utilizados na simulação do processo de soldagem. Entre eles, pode-se citar o ANSYS, utilizado neste trabalho, e o ABAQUS, que são programas com largo espectro de aplicações. Exemplos de programas comerciais específicos para a simulação do processo de soldagem são o SYSWELD e o SORPAS.

O calor gerado no RSW é o decorrente do efeito Joule, dependente das resistências elétricas no circuito secundário da máquina de solda, composto pelos eletrodos e pelas chapas. As resistências elétricas aí existentes são sete, mostradas na Figura 1: o eletrodo superior $\mathrm{R} 1$; a resistência de contato na interface deste eletrodo com a chapa R2; a chapa superior R3; a resistência de contato na interface entre as duas chapas R4; a chapa inferior R5; a resistência de contato na interface entre a chapa inferior e o eletrodo R6; o eletrodo inferior R7. As resistências elétricas de contato dependem do tipo de material, das rugosidades das superfícies, da pressão aplicada, da temperatura e da eventual presença de filmes de óxidos nas superfícies [8-10].

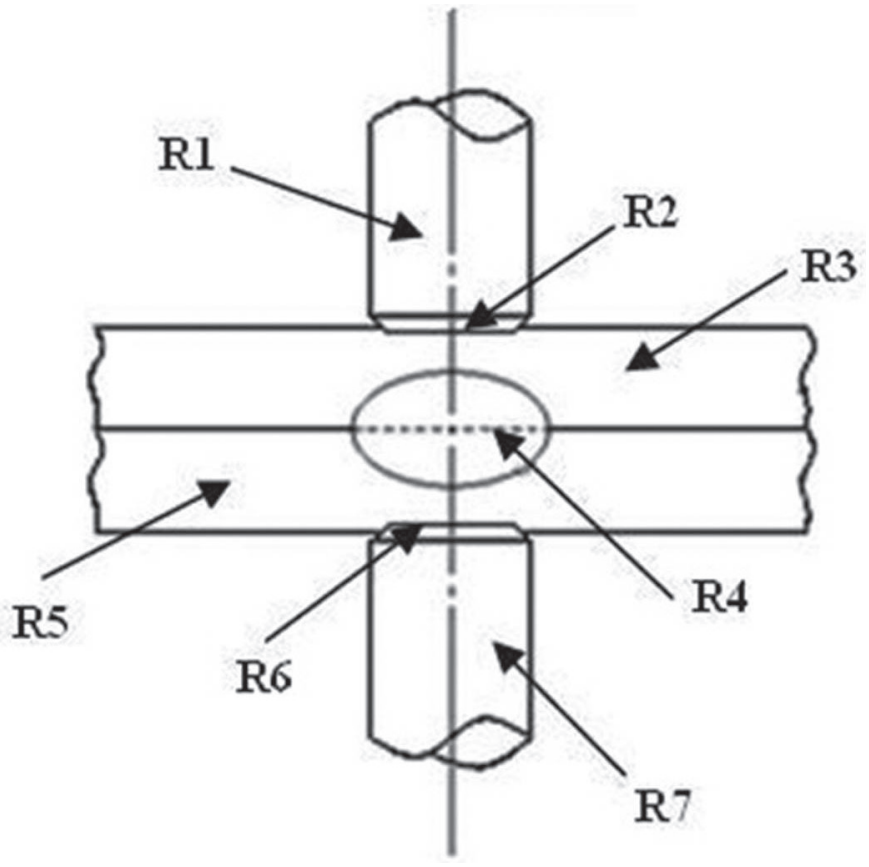

Figura 1. As resistências elétricas na solda ponto.

As variáveis de controle básicas no RSW são: a força de aperto dos eletrodos, o diâmetro da face do eletrodo, a corrente e o tempo de aplicação da corrente. Essas variáveis devem ser ajustadas de acordo com o material (ou materiais), as espessuras das chapas a serem soldadas e requisitos de qualidade da solda. A força de aperto dos eletrodos tem por finalidade a manutenção do contato entre os diferentes elementos no local da solda e influencia nas resistências elétricas e térmicas entre as duas chapas e entre as chapas e os eletrodos.
Na literatura é comum citar-se a resistência na interface entre as duas chapas, como sendo a resistência crítica no surgimento e crescimento da lentilha no RSW [8, 10, 11]. Essa resistência seria a maior entre todas, provocando então uma maior geração de calor e determinando o local da lentilha de solda. Neste trabalho, a força de aperto e as resistências elétricas e térmicas de contato não serão consideradas. Em consequiência, o modelo se afasta do processo real, mas pode contribuir para a determinação da importância relativa das diferentes variáveis intervenientes, pois permite determinar a geometria da lentilha de solda sem a deformação provocada pela aplicação de uma força de aperto nos eletrodos ou pela presença de resistências de contato. Além disso, evidências experimentais mostram que a resistência de contato entre as duas chapas desempenha alguma influência somente no início do processo, até o primeiro ou segundo ciclo de aplicação da corrente(dependendo do valor da corrente) [12]. Em seguida ela desaparece devido ao colapso (fusão) das asperezas inerentes à rugosidade das chapas. Também, no RSW aplicado a duas chapas com diferentes espessuras, realizado com os mesmos eletrodos dos dois lados, a lentilha gerada não é simétrica em relação à interface entre as duas chapas. Isto sugere que a resistência elétrica de contato entre as duas chapas não é a única resistência elétrica determinante da localização e geometria da lentilha, podendo ocorrer que, dependendo da configuração física (espessuras de chapas, materiais e resistências elétricas e térmicas de contato), sua importância seja menor que a resistência elétrica conjunta das duas chapas. Desse modo, com as simplificações consideradas, os parâmetros a serem variados na análise são o diâmetro da face do eletrodo, a corrente e o tempo de aplicação da corrente.

Este trabalho tem como objetivo obter informações a respeito da evolução, no tempo, da geometria da lentilha de solda em chapas de aço inoxidável austenítico AISI304. A geometria da lentilha de solda é caracterizada pela penetração e pelo diâmetro, mostrados na Figura 2. Essas duas dimensões são, entre outros, fatores que influenciam na qualidade do ponto de solda modificando sua resistência [10]. De acordo com Jou [13] a lentilha deve ter uma penetração de 0,6 a 0,7 vezes a espessura total da união. Já o diâmetro, em aplicações industriais, normalmente varia de $3 t^{1 / 2}$ a $6 t^{1 / 2}$, onde é a espessura da chapa em mm, segundo Zhou et al. [14]. De acordo com Machado [15], o diâmetro do ponto de solda fica aproximadamente $10 \%$ maior que o diâmetro da face do eletrodo e este usualmente é dado por $\mathbf{5} t \mathbf{1} / 2$. Deve-se observar que essas recomendações para a geometria do ponto de solda podem variar, de acordo com os requisitos de cada aplicação em particular. Os aços inoxidáveis austeníticos (série AISI 300) são importantes devido à possibilidade de uso na fabricação de equipamentos e tubos para as indústrias frigoríficas, alimentícias e químicas. As condições de soldagem desses aços diferem daquelas dos aços carbono comuns devido às suas diferentes propriedades físico-químicas, notadamente sua resistividade elétrica e difusividade térmica. As resistividades elétricas dos aços inoxidáveis variam entre quatro a dez vezes as dos aços comuns. O valor da difusividade térmica se situa entre um terço à metade da difusividade térmica dos aços comuns. Essas duas características sinalizam para o uso de uma menor corrente e um tempo menor para obtenção da solda. 


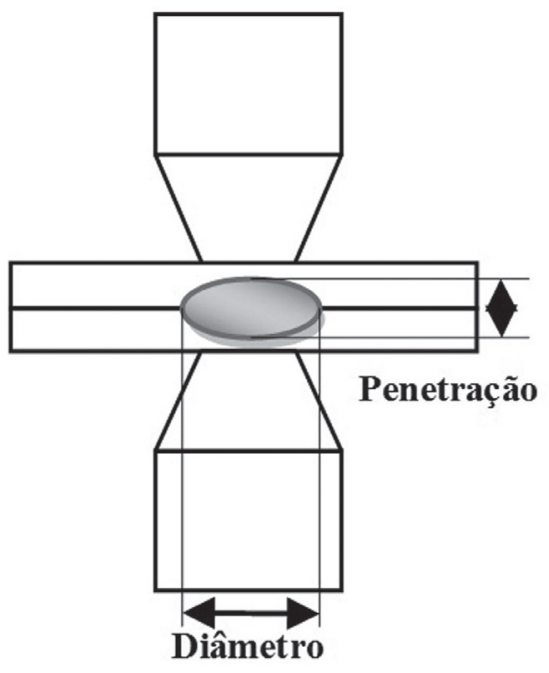

Figura 2. Geometria da lente da solda ponto.

\section{Modelo Matemático}

A geometria do problema que se deseja simular é apresentada na Figura 3, onde estão representados os dois eletrodos e as duas chapas em forma de discos. Foram realizadas algumas simplificações com intuito de reduzir o tempo de simulação. A análise transiente do processo de solda ponto foi modelada como um modelo axissimétrico e bidimensional no software ANSYS. Como o modelo é simétrico em relação à superfície de contato entre as duas chapas, somente a metade superior será considerada. O problema envolve acoplamento entre os campos elétrico e térmico porque a distribuição de temperatura afeta a resistividade. Esta altera a distribuição de densidade de corrente, que por sua vez altera a taxa de geração de calor por efeito Joule nos diferentes pontos do domínio de cálculo, o que afeta a distribuição de temperatura.

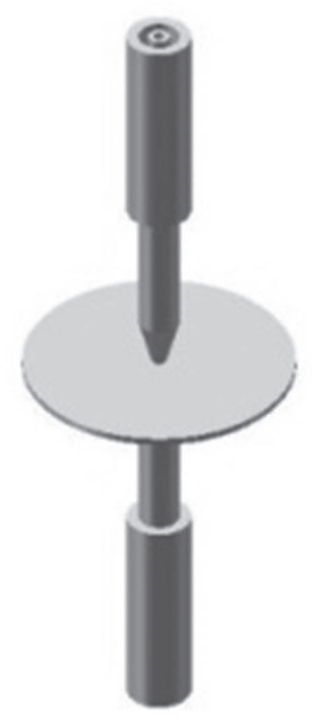

Figura 3. Solda ponto em chapas com formato de discos

\subsection{Equações diferenciais}

O campo elétrico se estabelece em um intervalo de tempo muito menor que o térmico de tal forma que é possível utilizar para a análise elétrica a equação de Laplace, em termos do potencial elétrico $f$ e da resistividade elétrica $\rho_{e}$. Deve-se notar que devido à dependência da resistividade elétrica em relação à temperatura, essa equação deve ser resolvida em cada passo no tempo, já que o problema térmico é transiente.

$\boldsymbol{\nabla} \cdot\left(\frac{1}{\rho_{e}} \nabla \phi\right)=\mathbf{0}$

A densidade de corrente, $I$ e o calor gerado por unidade de volume e tempo, $Q$, devido ao efeito Joule podem ser determinados por:

$J=-\frac{1}{\rho_{e}} \nabla \phi$

$Q=\frac{1}{\rho_{e}}(\nabla \phi \cdot \nabla \phi)$

Para tratar problemas de condução de calor onde ocorrem mudanças de estado (de sólido para líquido, por exemplo), uma das alternativas é a utilização da equação para condução de calor em regime transiente com geração de calor, formulada em termos da função entalpia [16], conforme a equação (4).

$\frac{d H}{d t}=\boldsymbol{\nabla} \cdot(k \nabla T)+Q$

Onde $H$ é a função entalpia por unidade de volume e $k$ é a condutividade térmica, ambas funções da temperatura $T$. Para evitar problemas numéricos é considerado que a mudança de estado, de sólido para líquido, ocorre em um pequeno intervalo de temperaturas, de $T s$ até $T_{l}$, como mostra a Figura 4.

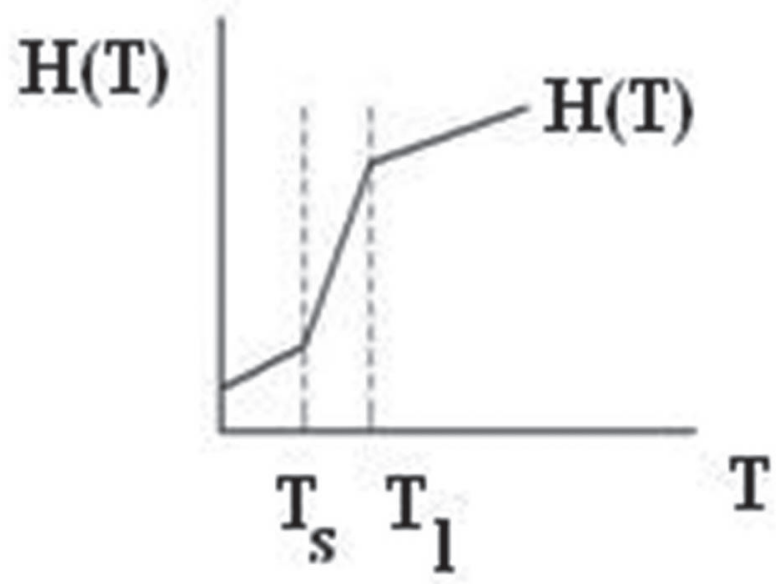

Figura 4. Variação da entalpia com a temperatura. 


\subsection{Condições de contorno}

Devem ser consideradas as condições de contorno tanto para o campo elétrico como para o térmico. Primeiramente são definidas aquelas para o campo elétrico. Nas superfícies da chapa e do eletrodo em contato com o ar coloca-se a seguinte condição de contorno:

$\frac{\partial \phi}{\partial n}=\mathbf{0}$

Onde $n$ representa a normal à superfície considerada.

Já as condições de contorno para o topo e a base do conjunto são respectivamente: potencial conhecido e potencial nulo. Estas condições de contorno estão representadas na Figura 5.

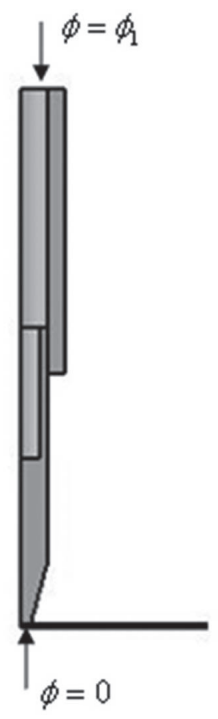

Figura 5. Condições de contorno para o campo elétrico, no topo e na interface entre as chapas.

Para o campo térmico têm-se como condições de contorno a convecção natural para as partes expostas ao ar e convecção interna forçada para as partes em contato com a água. A Figura 6 ilustra estas condições de contorno. As outras superfícies são consideradas isoladas termicamente.

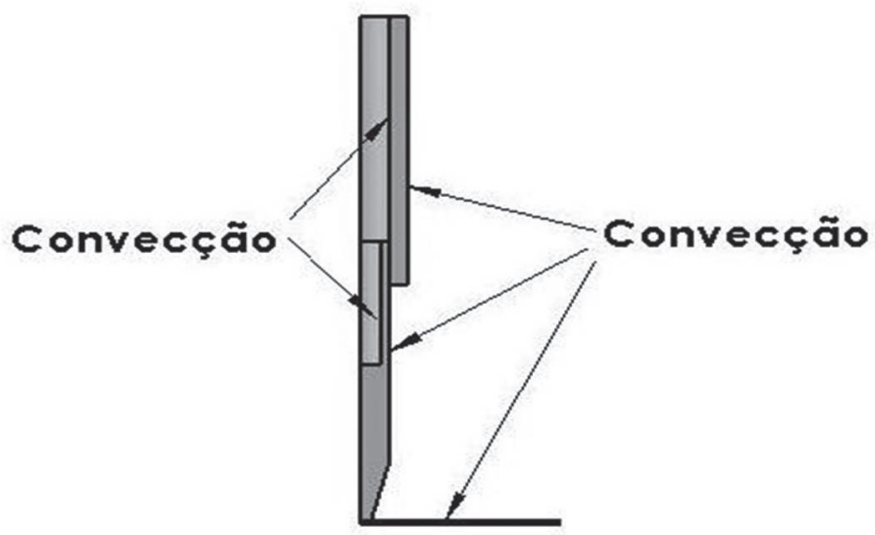

Figura 6. Condições de contorno para o campo térmico

\subsection{Os coeficientes de troca de calor por convecção}

Na Figura 7 estão enumeradas as superfícies onde são calculados os coeficientes de transferência de calor por convecção. Para fins de exemplo de valores de coeficientes de transferência de calor por convecção utilizados são apresentados os resultados referentes a um dos eletrodos usados nas simulações, o eletrodo 1.

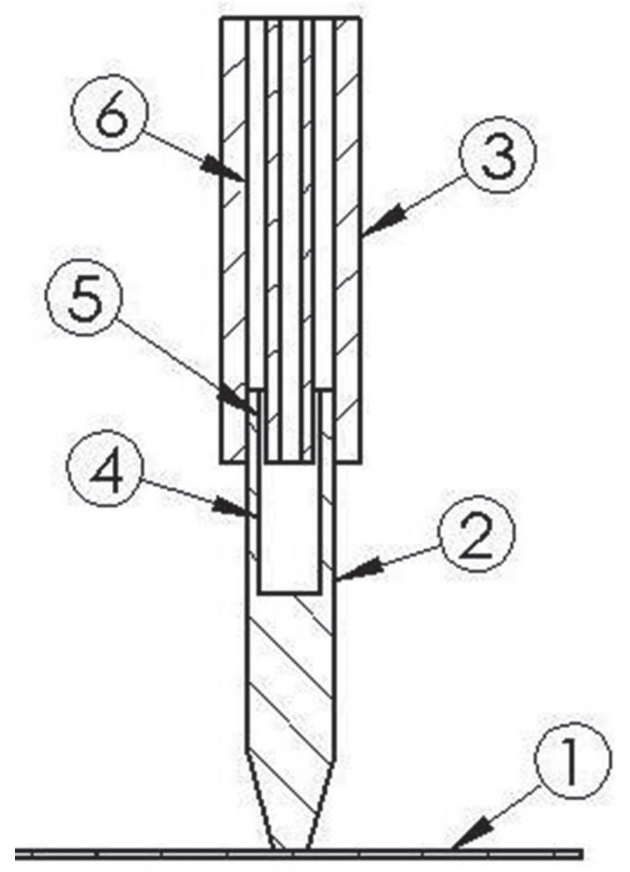

Figura 7. Superfícies onde são necessários os coeficientes de transferência de calor por convecção.

\subsubsection{Convecção natural na superfície 1}

Conforme Bejan [17], o número de Nusselt médio, $\overline{N u}_{L}$, para a convecção natural numa placa horizontal aquecida é dependente do número de Rayleigh, $R a_{L}$, através da seguinte correlação:

$$
\overline{N u}_{L}=0,54 R a_{L}^{1 / 4} \quad\left(10^{4}<R a_{L}<10^{7}\right)
$$

Onde; $R a_{L}=g \beta\left(T_{w}-T_{\infty}\right) L^{3} / \alpha v ; \beta=\mathbf{1} / T$ é o coeficiente de expansã̃o térmica; $g$ é a aceleração da gravidade, Tw é a temperatura da superfície; é a temperatura do fluido afastado da superfície; L é o comprimento característico da placa. Para um ${ }^{\text {disco }} L=\frac{D}{4}$. a, o diâmetro, foi arbitrado para fins de simulação igual a $0,2 \mathrm{~m}$. é a difusividade térmica e $v$ é a viscosidade cinemática do fluído. Os valores das propriedades do ar devem ser obtidos na temperatura de filme $T_{f}=\frac{T_{w}+T_{\infty}}{2}=1073,15 \mathrm{~K}+298,15 \mathrm{~K}=686,15 \mathrm{~K} \cdot$ O uso da Equação (6) resultou num coeficiente de troca de calor por convecção médio . 


\subsubsection{Convecção natural nas superfícies $\mathbf{2}$ e 3}

De acordo com Bejan [17], um cilindro vertical pode ser considerado como uma parede vertical se a espessura da camada limite for muito menor que o diâmetro do cilindro. Neste caso a curvatura da superfície lateral não afeta significativamente o processo de transferência de calor. Após verificação que as superfícies 2 e 3 mostradas na Figura 7 atendem a esse critério, a determinação do Nusselt médio foi realizada com a seguinte correlação [17]:

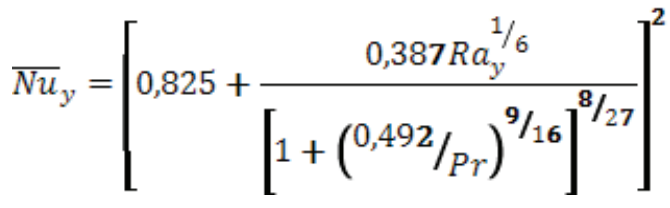

Onde Prīo o número de Prandt e $y$ a distância a partir da base da parede. $\mathrm{O}$ uso da Equação (7) resultou num coeficiente de troca de calor por convecção médio $\quad \overline{\boldsymbol{h}}=14,6 \mathrm{~W} \cdot \mathrm{m}^{-2} \cdot \mathrm{K}^{-1}$ para a superfície 2 e $\overline{\boldsymbol{h}}=11,0 \mathrm{~W} \cdot \mathrm{m}^{-2} \cdot \mathrm{K}^{-\mathbf{1}}$ para a superfície 3 .

\subsubsection{Convecção forçada na superfície 4}

Nas superfícies 4, 5 e 6, apresentadas na Figura 7 o eletrodo é resfriado com água. Conforme Aures [18] a vazão de água recomendada para soldar chapas de aço sem revestimento e com espessura de até $3 \mathrm{~mm}$ é de 4 1/min. Para chapas de aço com revestimento e/ou grande espessura deve ser de 6 a 7 1/min. Neste trabalho foi adotada uma vazão de 5 1/min.

A troca de calor na superfície 4, na falta de um estudo mais preciso, é aproximada como convecção no interior de um tubo com temperatura de parede uniforme. Para essa situação, conforme Bejan [17], no cálculo do coeficiente de troca de calor por convecção, pode-se utilizar a seguinte correlação para o Nusselt:

$$
\begin{aligned}
N u_{D}= & 0,012\left(R e_{D}^{0,87}-280\right) \operatorname{Pr}^{0,4} \\
& \left(1,5 \leq \operatorname{Pr} \leq 500 \text { e } 3 \times 10^{3} \leq R e_{D} \leq 10^{6}\right)
\end{aligned}
$$

Onde $R e_{D}$ é o número de Reynolds calculado com o diâmetro $D$ do tubo. $\mathrm{O}$ valor do coeficiente de troca de calor por convecção determinado foi $\boldsymbol{h}=8322 \mathrm{~W} \cdot \mathrm{m}^{-2} \cdot \mathrm{K}^{-\mathbf{1}}$.

\subsubsection{Convecção forçada nas superfícies 5 e 6}

Os escoamentos nas superfícies 5 e 6 ocorrem numa região anular entre tubos concêntricos. Segundo Incropera [19], para uma primeira aproximação, os coeficientes de convecção, interno e externo, podem ser considerados iguais, e podem ser avaliados utilizando-se o diâmetro hidráulico, com a mesma Equação (8). $\mathrm{O}$ que resultou num coeficiente de troca de calor por convecção $\boldsymbol{h}=21870 \mathrm{~W} \cdot \mathrm{m}^{-2} \cdot \mathrm{K}^{-\mathbf{1}}$ para a superfície 5 $\boldsymbol{h}=5897 \mathrm{~W} \cdot \mathrm{m}^{-2} \cdot K^{-1} \quad$ e para a superfície 6 .

\subsection{Propriedades dos materiais}

O calor específico e a condutividade térmica do aço inox AISI 304 em diferentes temperaturas foram obtidos na literatura [17,19]. A fusão do AISI 304 ocorre na faixa de temperaturas entre $1673,15 \mathrm{~K}$ e $1728,15 \mathrm{~K}$ [20]. A resistividade elétrica do AISI 304 é também variável com a temperatura. Foram encontrados valores dessa propriedade em apenas duas temperaturas [20], utilizando-se então interpolação ou extrapolação linear para temperaturas diferentes.

O eletrodo segue a norma AWS D8.6, grupo A, classe 2 (99,2\% $\mathrm{Cu} ; 0,8 \% \mathrm{Cr}$ ). O ideal seria considerar no modelo matemático que as propriedades dos eletrodos são também dependentes da temperatura. No entanto, não foram obtidos dados a respeito dessa dependência de forma que suas propriedades térmicas e elétricas são consideradas constantes conforme a Tabela 1[21].

Tabela 1. Propriedades do eletrodo [21

\begin{tabular}{|l|c|l|}
\hline Densidade & & $8820 \mathrm{~kg} / \mathrm{m}^{3}$ \\
\hline Condutividade térmica & $\mathrm{k}$ & $315 \mathrm{~W} / \mathrm{m} . \mathrm{K}$ \\
\hline Calor específico & $\mathrm{c}$ & $376 \mathrm{~J} / \mathrm{kg} . \mathrm{K}$ \\
\hline Resistividade elétrica & $\mathrm{e}$ & $3,83 \times 10^{-8} \Omega . \mathrm{m}$ \\
\hline
\end{tabular}

\section{Simulações}

Foram realizadas simulações considerando chapas com espessura de $1 \mathrm{~mm}, 2 \mathrm{~mm}$ e $3 \mathrm{~mm}$, utilizando-se dois eletrodos diferentes. Devido ao fato que as resistências elétricas são alteradas quando uma determinada corrente passa através de áreas diferentes de chapas, foram considerados vários diâmetros de face de eletrodo com a finalidade de verificar a influência desse parâmetro sobre o tempo de formação e a geometria da lentilha de solda. O eletrodo 1, apresentado na Figura 8, com diâmetros de face de $3,5 \mathrm{~mm}, 4,0 \mathrm{~mm}$ e $4,5 \mathrm{~mm}$ foi usado nas simulações de soldagem de chapas de $1 \mathrm{~mm}$. O eletrodo 2 , apresentado na Figura 9, com diâmetros de face de $6 \mathrm{~mm}, 7$ $\mathrm{mm}, 8,5 \mathrm{~mm}, 10 \mathrm{~mm}$ e $11 \mathrm{~mm}$ foi utilizado nas simulações de soldagem de chapas de $2 \mathrm{~mm}$. O mesmo eletrodo 2, mas com diâmetros de face de $10 \mathrm{~mm}, 10,5 \mathrm{~mm}, 12,75 \mathrm{~mm}$ e $15 \mathrm{~mm}$, foi utilizado nas simulações referentes às chapas de $3 \mathrm{~mm}$. Para cada configuração foram simulados diferentes valores de condições de contorno elétricas e verificado o tempo e a corrente na qual eram atingidos os valores recomendados do diâmetro e da penetração da lente de solda.A Figura 10 mostra a sequiência lógica de análise. A partir do modelo físico, são verificadas simetrias que permitem a simplificação da geometria do problema. No modelo simplificado é gerado a malha, aplicadas as condições iniciais e de contorno e como resultado das simulações, obtémse a evolução no tempo do campo de temperaturas. Nessa figura, para melhor visualização da malha e do campo de temperaturas, é mostrada somente parte do eletrodo e da chapa. 

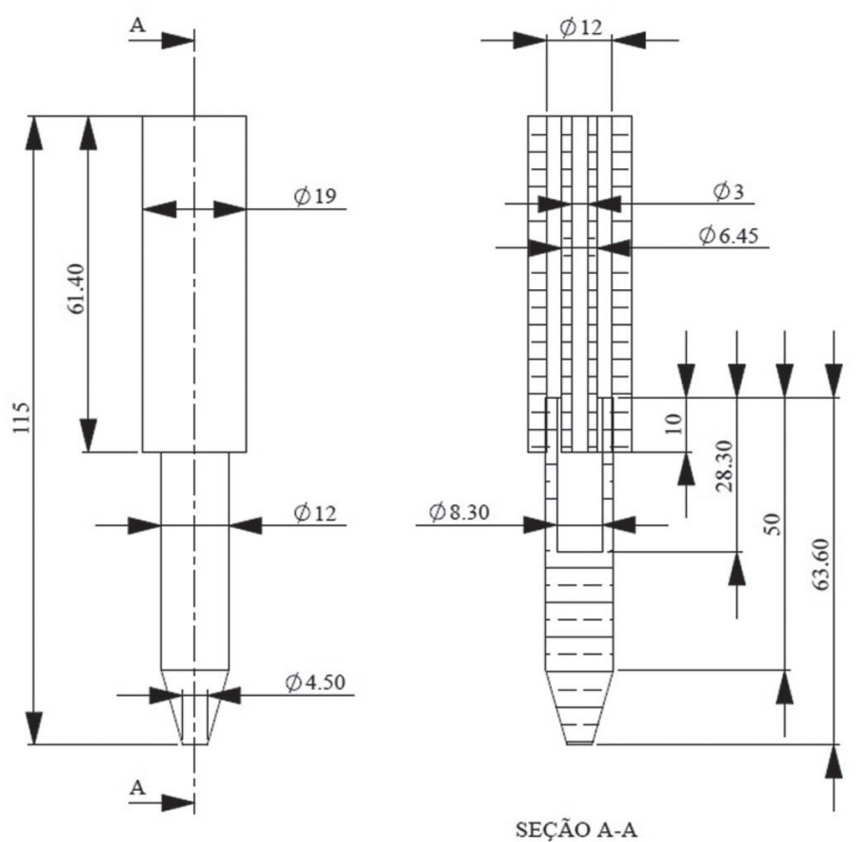

Figura 8. Dimensões do eletrodo 1 em milímetros.
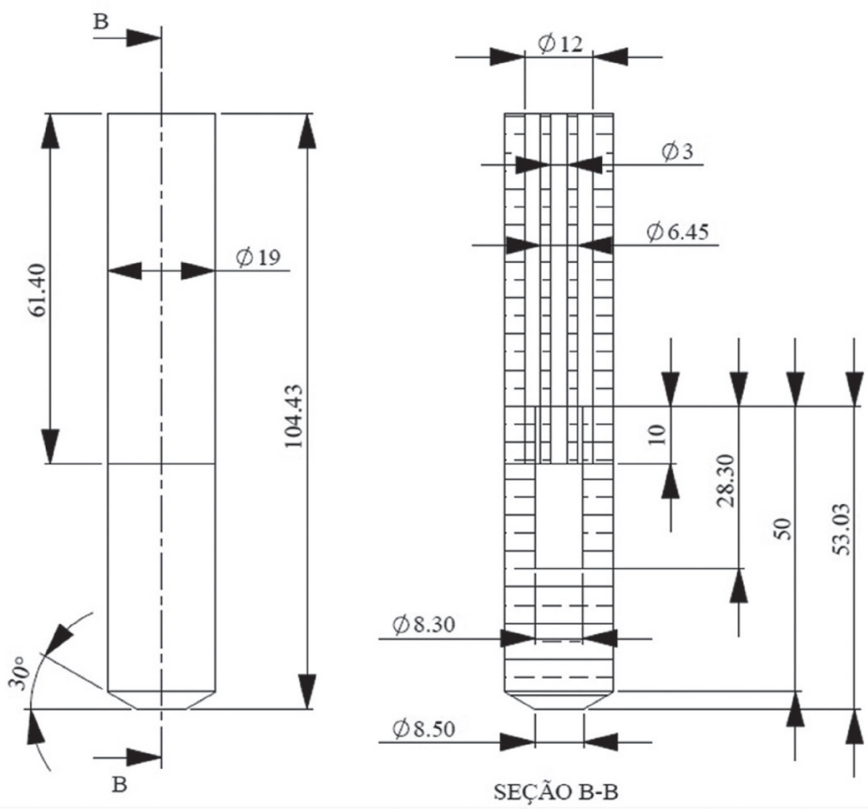

Figura 9. Dimensões do eletrodo 2 em milímetros.

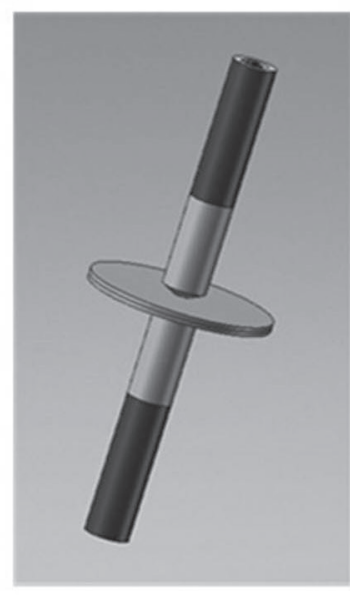

(a)

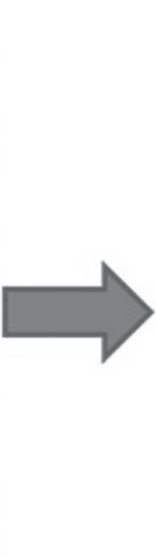

(b)
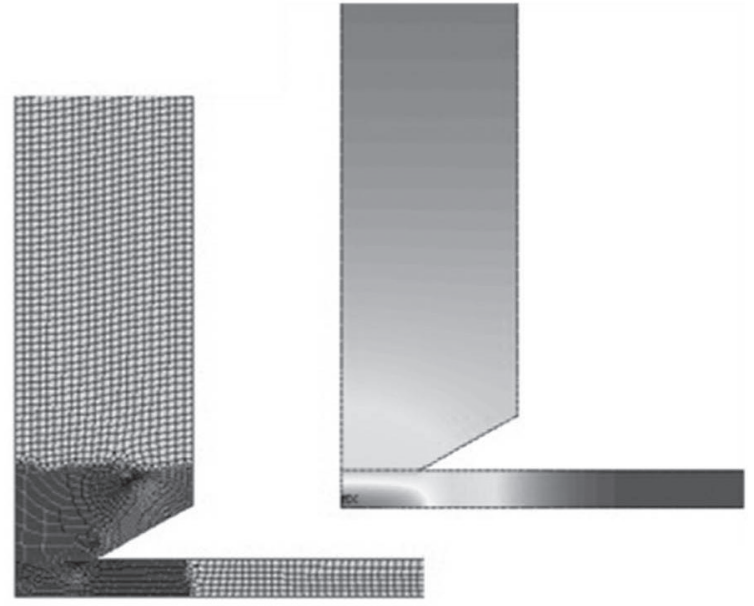

Figura 10. Modelagem do problema no software ANSYS. (a) Modelo físico; (b) modelo bidimensional axissimétrico da ponta do eletrodo e parte da chapa; (c) malha de elementos finitos, (d) campo de temperaturas.

A implementação no programa Ansys foi realizada utilizando o elemento Plane 67 que possue capacidade de condução térmica, condução elétrica, e inclui, no balanço de energia, o calor gerado pelo efeito Joule. Como este elemento permite, foi adotado o método direto de solução do problema acoplado, considerando todas as variáveis na mesma análise. Foram realizados testes de sensibilidade de malha e de passo no tempo, resultando na utilização de uma malha com 8673 elementos para eletrodo 1, 7661 elementos para o eletrodo 2, e um passo no tempo de , permitindo que o programa reduza automaticamente o passo no tempo, quando necessário para a convergência.

\section{Resultados}

A Figura 11 mostra um exemplo de campo de temperaturas obtido nas simulações. A partir desses resultados foram determinadas as distribuições de temperatura. Temperaturas iguais ou maiores que a temperatura de fusão, $1673 \mathrm{~K}$, determinam a geometria da lentilha de solda. 


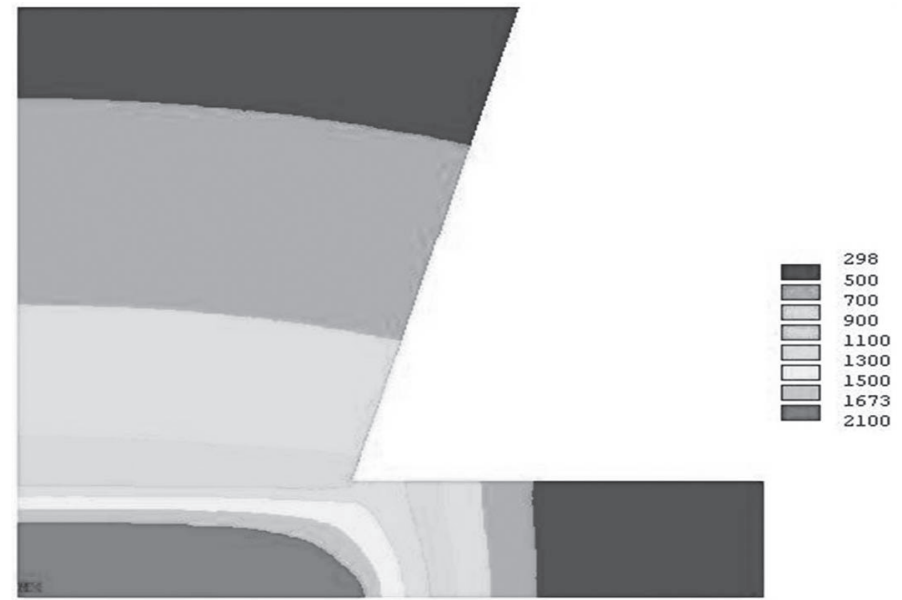

Figura 11. Distribuição de temperaturas (K). Chapa: $1 \mathrm{~mm}$; corrente: $5400 \mathrm{~A}$; tempo: $0,12 \mathrm{~s}$.

\subsection{Comparação com resultados experimentais}

Para avaliação do modelo utilizado neste trabalho, foi realizada uma pesquisa, não exaustiva, na literatura, por resultados experimentais referentes ao aço AISI $304 \mathrm{e}$, na falta destes, trabalhos com outros aços. Nos trabalhos encontrados foi verificado que não havia uma completa descrição da metodologia experimental com especificação do sistema de controle e medida da corrente elétrica e estimativa dos erros experimentais na medida da corrente, do diâmetro da lentilha de solda, penetração, diâmetro da face do eletrodo, alinhamento dos eletrodos, força aplicada. Além disso, os resultados apresentados se referem normalmente a pontos com uma única amostra, não havendo preocupação com a repetibilidade das medidas. Também, não foram encontrados trabalhos independentes tratando das mesmas condições experimentais para confirmação dos resultados.

Foram encontrados alguns poucos trabalhos usando o a aço AISI 304. Karci et al. [3] apresentam a variação do tamanho da lentilha de solda em função do tempo de aplicação da corrente, em chapas de aço de $2 \mathrm{~mm}$ de espessura, usando um eletrodo com diâmetro de face de $8 \mathrm{~mm}$, mas não apresentam o valor da corrente utilizada. Vural et al. [22] apresentam medidas do diâmetro da lentilha de solda, em chapas de 1,03 $\mathrm{mm}$ de espessura, em função da corrente aplicada durante um tempo referente a 15 períodos (frequiência de $50 \mathrm{~Hz}$ ), mas não apresentam o valor do diâmetro da face do eletrodo utilizado. Marashi et al. [23] apresentam a variação do diâmetro da lentilha de solda em função da corrente para um tempo equivalente a 12 ciclos (freqüência de $50 \mathrm{~Hz}$ ), em chapas de 1,2 $\mathrm{mm}$ de espessura e com um eletrodo com diâmetro de face igual a $7 \mathrm{~mm}$. A Figura 12 apresenta a comparação entre os resultados obtidos pelas simulações com os resultados de Marashi et al.[23].

Não foi encontrado nenhum trabalho experimental com o aço AISI 304 que apresentasse a variação da penetração da lentilha de solda em função da corrente ou do tempo de aplicação de uma determinada corrente. Para o aço AISI 1008 Gould [24] apresenta medidas da penetração da lentilha de solda em função da corrente aplicada para um tempo relativo a 15 ciclos (frequiência de $60 \mathrm{~Hz}$ ), em chapas de 1,09 mm de espessura, com eletrodos de diâmetro de $6,1 \mathrm{~mm}$. A Figura 13 mostra a comparação dos resultados obtidos nas simulações com os apresentados por Gould [24]. Os dados referentes às propriedades elétricas e térmicas do aço AISI 1008 usados nas simulações foram obtidos na referência [25].

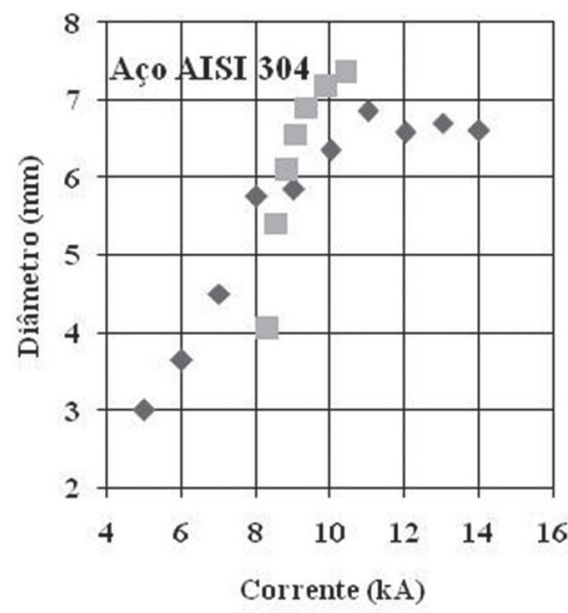

\section{$\bullet$ Resultados experimentais de Marashi}

Wimulaçōes

Figura 12. Comparação entre resultados das simulações e resultados experimentais de Marashi et al. [23].

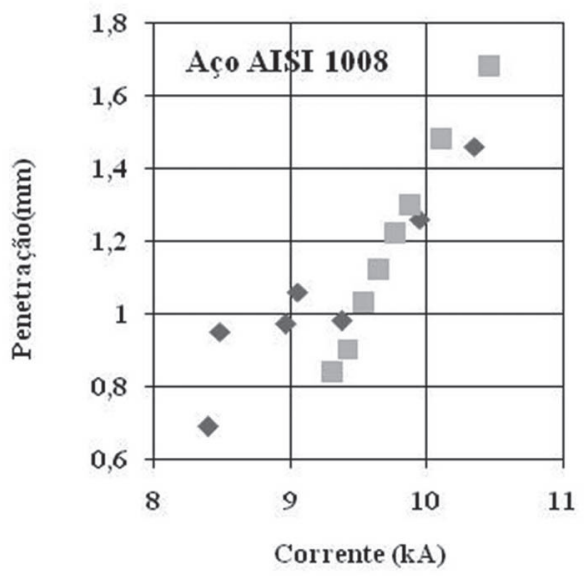

Resultados Experimentais de Gould

Simulaçães

Figura 13. Comparação entre resultados das simulações e resultados experimentais de Gould [24].

Observa-se nas Figuras 12 e 13 que a concordância entre os resultados das simulações com os resultados experimentais é dependente da faixa de valores da corrente, sendo reduzida em valores de correntes menores. A causa dessa baixa concordância nessa faixa de corrente deve ainda ser investigada.

\subsection{Resultados para as chapas de $1 \mathrm{~mm}, 2 \mathrm{~mm}$ e $3 \mathrm{~mm}$}

Para as chapas de espessura de $1 \mathrm{~mm}$, a penetração deve estar entre 1,2 mm e 1,4 mm [13] e o diâmetro da lentilha de solda entre $3,5 \mathrm{~mm}$ e $5 \mathrm{~mm}$ [14]. Para a apresentação dos resultados, na Figura 14, foi escolhido fixar o valor da penetração em 1,2 $\mathrm{mm}$, de forma a facilitar a análise. Nessa figura, os valores da corrente são aqueles calculados no instante de tempo do resultado apresentado, pois como as condições de contorno 
aplicadas para determinação do campo elétrico são tensões, devido ao aumento da resistividade elétrica com a temperatura as correntes apresentam uma diminuição à medida que as chapas são aquecidas.

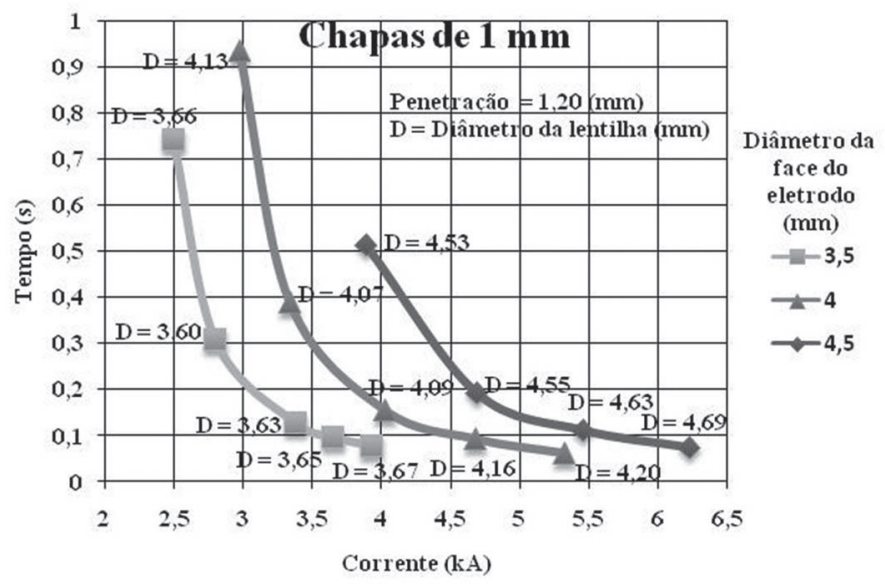

Figura 14. Tempo de soldagem em função da corrente para chapas de $1 \mathrm{~mm}$.

Para as chapas de espessura de $2 \mathrm{~mm}$, o diâmetro da lentilha de solda deve estar entre $7 \mathrm{~mm}$ e $10 \mathrm{~mm}$ e a penetração entre $2,4 \mathrm{~mm}$ e $2,8 \mathrm{~mm}$. Simulações realizadas com o eletrodo 1 , diâmetro de face $4,5 \mathrm{~mm}$, permitiram verificar que este eletrodo é inadequado para essa espessura de chapa. Quando o diâmetro da lentilha de solda se aproximava do valor mínimo a penetração ultrapassava em muito o valor máximo, aproximando-se da espessura das duas chapas juntas, o que pode provocar uma grande indentação e expulsão. Os resultados das simulações realizadas com o eletrodo 2 são mostrados na Figura 15, onde o valor da penetração foi fixado em 2,40 mm. Para o eletrodo com diâmetro de face $6 \mathrm{~mm}$ não foi atingido o valor mínimo de diâmetro da lentilha de solda, para essa penetração e dentro das faixas de corrente e tempo utilizadas nas simulações. Dessa forma, nas aplicações onde é exigido que o diâmetro da lentilha tenha um valor mínimo de $7 \mathrm{~mm}$, este diâmetro de face não é recomendado, pois para atingir esse valor, seria necessário aceitar uma maior penetração, o que, dependendo da aplicação, pode não ser desejável. Os resultados também mostram que com o uso dos eletrodos com diâmetro de face de $10 \mathrm{~mm}$ e $11 \mathrm{~mm}$ são obtidos diâmetros das lentilhas de solda maiores que o valor máximo do intervalo recomendado, para valores de penetração mínimos, o que pode torná-los indesejáveis para algumas aplicações.

Para as chapas de espessura $3 \mathrm{~mm}$, o diâmetro da lentilha de solda deve estar entre $10,5 \mathrm{~mm}$ e $15 \mathrm{~mm}$ e a penetração entre $3,6 \mathrm{~mm}$ e 4,2 mm. Os diâmetros de face de eletrodos testados foram de: $10 \mathrm{~mm}, 10,5 \mathrm{~mm}, 12,75 \mathrm{~mm}$ e $15 \mathrm{~mm}$. Os resultados são apresentados na Figura 16 onde a penetração foi fixada em $3,60 \mathrm{~mm}$. Para o eletrodo com face de diâmetro igual a $15 \mathrm{~mm}$, os diâmetros da lentilha ficaram em torno do valor máximo recomendado enquanto a penetração estava em seu valor mínimo.

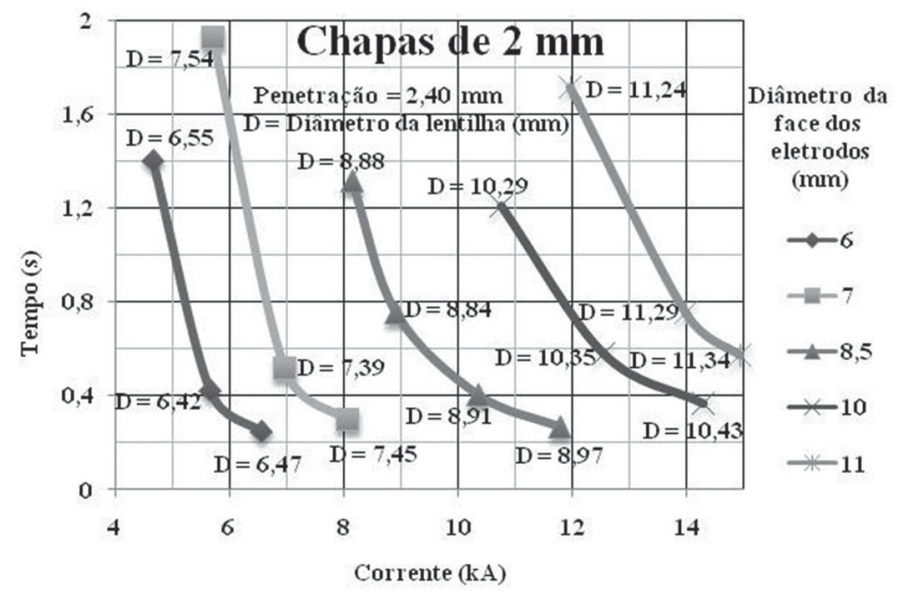

Figura 15. Tempo de soldagem em função da corrente para chapas de $2 \mathrm{~mm}$.

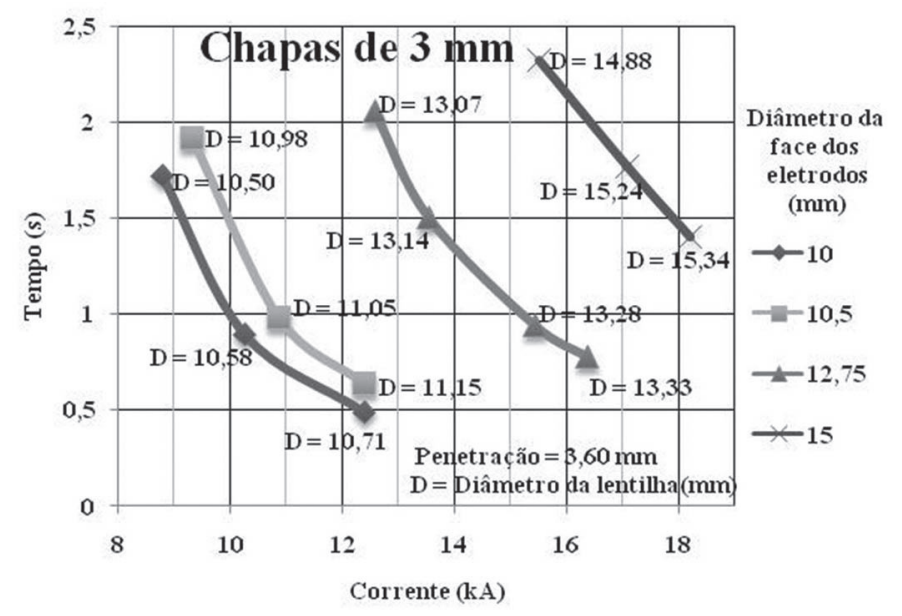

Figura 16. Tempo de soldagem em função da corrente para as chapas de $3 \mathrm{~mm}$.

Da análise das Figuras 14, 15 e 16, verifica-se que para um mesmo valor de corrente, um eletrodo com diâmetro de face maior produz uma lentilha de solda com maior diâmetro, mas exige um maior tempo de aplicação da corrente. Ou, invertendo a análise, para um mesmo tempo de aplicação da corrente, o uso de um eletrodo com diâmetro de face maior, produz uma lentilha de solda com diâmetro maior, mas exige uma corrente maior.

$\mathrm{Na}$ Figura 14, nas curvas referentes aos eletrodos com diâmetros de face $3,5 \mathrm{~mm}$ e $4 \mathrm{~mm}$, e na Figura 15, nas curvas referentes aos eletrodos com diâmetros de face $6 \mathrm{~mm}, 7 \mathrm{~mm}$ e 8,5 $\mathrm{mm}$, observa-se que, inicialmente, o diâmetro da lentilha de solda decresce com o aumento da corrente e após apresenta tendência contrária. Uma possível explicação para esse comportamento é que com baixos valores de corrente, baixas taxas de geração de calor, a transferência de calor radial favorece o crescimento do diâmetro da lentilha de solda. Com o aumento da corrente ocorre um aumento da taxa de geração de calor no eixo da lentilha de solda de maneira a favorecer o crescimento da penetração em relação ao diâmetro. A partir de determinado valor de corrente o diâmetro da lentilha de solda começa a aumentar com o aumento da corrente. Isso é explicado pelo aumento da resistividade 
elétrica com a temperatura. A corrente elétrica percorre, entre dois pontos, o trajeto de menor resistência. Devido ao aumento da temperatura, na região central da lentilha de solda, ocorre uma diminuição da densidade de corrente nessa região em favor de aumento nas regiões próximas, de menor temperatura. Em altos valores de corrente, a fração desviada de corrente pode ser suficiente para favorecer o aumento do diâmetro da lentilha de solda.

A partir dos valores de corrente obtidos e o seu tempo de aplicação é possível a determinação da energia consumida no processo de soldagem para cada valor de diferença de tensão aplicado. Os resultados, apresentados na Figura 17, indicam que quanto maiores as correntes, aplicadas durante um tempo menor, menos energia é consumida. Isto evidencia que a taxa de perda de calor da região de soldagem não consegue acompanhar o aumento da taxa de geração de calor devido ao aumento da corrente. É importante ressaltar que esta conclusão é limitada à energia consumida na formação da lentilha. Não é referente ao processo global que deve incluir as perdas da máquina de solda, variáveis com a corrente.

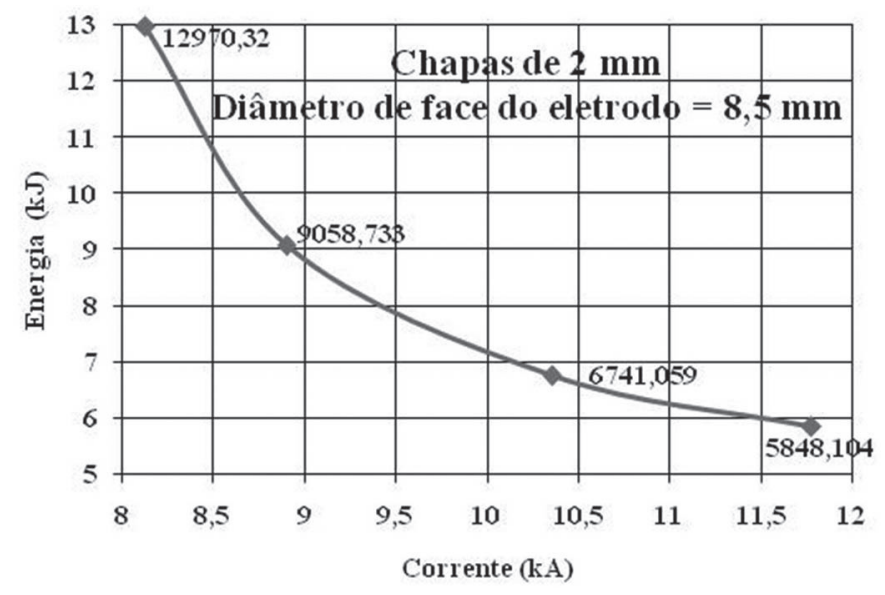

Figura 17. Energia consumida durante o processo de soldagem.

\section{Conclusões}

Este trabalho apresentou simulações numéricas do crescimento da lentilha de solda obtida pelo processo de soldagem a ponto por resistência elétrica em chapas de aço inoxidável AISI 304. Foi desenvolvido um modelo bidimensional axissimétrico e utilizado o método de elementos finitos em regime transiente. As propriedades elétricas e térmicas das chapas foram consideradas dependentes da temperatura. Foram considerados contatos perfeitos entre as duas chapas e entre as chapas e eletrodos e foi desconsiderada a força de aperto dos eletrodos.

Com esse modelo foi possível determinar a geometria da lentilha de solda, sem a deformação que seria produzida por uma força de aperto dos eletrodos e sem a influência das resistências elétricas e térmicas de contato. A comparação dos resultados das simulações com resultados experimentais obtidos na literatura evidencia uma concordância dependente da faixa de corrente, sendo reduzida em valores de menor corrente. Os resultados obtidos com as simulações indicam que o uso de eletrodos com diâmetro de face maior produz uma lentilha de solda com maior diâmetro, mas exige um maior tempo de aplicação da corrente. Foi verificado que para um mesmo eletrodo, comparando-se os resultados obtidos considerando o mesmo valor de penetração, ocorre uma faixa de correntes onde o diâmetro da lentilha de solda diminui com o aumento da corrente. Após, numa faixa de correntes maiores, o diâmetro aumenta com o aumento da corrente. Os resultados também mostram que com o uso de maiores correntes é consumida menos energia para formação da lentilha de solda ponto. Trabalhos futuros devem incluir no modelo resistências elétricas e térmicas de contato e força de aperto a fim de evidenciar a sua influência. Também é sugerida a análise do RSW considerando-se diferentes formatos de ponta do eletrodo.

\section{Referências Bibliográficas}

[1] SUN, X.; STEPHENS, E. V.; KHALEEL, M. A.; SHAO, H.; KIMCHI, M. Resistance Spot Welding of Aluminum Alloy to Steel with Transition Material - From Process to Performance - Part I: Experimental Study, Welding Journal, USA, v.83, nº.6, p.188s-195s, June. 2004.

[2] TAN, J. C.; WESTGATE, S. A.; CLYNE, T. W. Resistance Welding of Thin Stainless Steel Sandwich Sheets with Fibrous Metallic Cores: Experimental and Numerical Studies, Science and Technology of Welding and Joining, UK, v. 12, $\mathrm{n}^{\circ}$ 6, p.490$504,2007$.

[3] KARCI, F.; KAÇAR, R.; GÜNDÜZ, S. The Effect of Process Parameter on the Properties of spot Welded Cold Deformed AISI 304 Grade Austenitic Stainless Steel, J. Mater. Process. Tech., v. 209, no 8, p. 4011-4019, April 2009.

[4] XU, L.; KHAN, J. A. Nugget Growth Model for Aluminum Alloys During Resistance Spot Welding, Welding Journal, USA, v. 78, no 11, p. 367s-372s, November 1999.

[5] SUN, X.; KHALEEL, M. A. Resistance Spot Welding of Aluminum Alloy to Steel with Transition Material - Part II: Finite Element Analyses of Nugget Growth, Welding Journal, USA, v.83, n.7, p.197S-202S, Jul. 2004.

[6] ZHIGANG, H.; KIM, I. S.; SON, J. S.; KIM, H. H.; SEO, J. H.; JANG, K. C.; LEE, D. K.; KUK, J. M. A Study on Numerical Analysis of the Resistance Spot Welding Process, Journal of Achievements in Materials and Manufacturing Engineering, v. 14, no 1-2, p. 140-145, January-February 2006.

[7] ZHANG, Y. S.; XU, J.; LAI, X. M.; CHEN, G. L. Numerical Simulation of Spot Welding for Galvanised Sheet Steels, Science and Technology of Welding and Joining, v. 13, n 2, p. 192-198, February 2008.

[8] SONG, Q.; ZHANG, W.; BAY, N. An Experimental Study Determines the Electrical Contact Resistance in Resistance Welding, Welding Journal, USA, v.84, n.5, p.73s-76s, May 2005. [9] BABU, S. S.; SANTELLA, M. L.; FENG, Z.; RIEMER, B. W.; COHRON, J. W. Empirical Model of Effects of Pressure and Temperature on Electrical Contact Resistance of Metals, Science and Technology of Welding \& Joining, v.6, n.3, p.126132, Jun. 2001. 
[10] RESISTANCE WELDING MANUAL, 4 ed. rev., RWMA, Philadelphia,2003. 472 p.

[11] ASLANLAR, S. The Effect of Nucleus Size on Mechanical Properties in Electrical Resistance Spot Welding of Sheets Used in Automotive Industry, Materials and Design, v. 27, $\mathrm{n}^{\circ}$ 2, $\mathrm{p}$. 125-131, 2006.

[12] CHO, Y.; RHEE, S. Experimental Study of Nugget Formation in Resistance Spot Welding, Welding Journal, USA, v.82 n.8, p.195s-201s, Aug. 2003.

[13] JOU, M. Real Time Monitoring Weld Quality of Resistance Spot Welding for the Fabrication of Sheet Metal Assemblies, Journal of Materials Processing Technology, v. 132, $\mathrm{n}^{\circ} 1-3$, p. 102-113, January 2003.

[14] ZHOU, M.; ZHANG, H.; HU, S. J. Relationship Between Quality and Attributes of Spot Welds, Welding Journal, USA, v. 82, n 4, p. 72s-77s, April 2003.

[15] MACHADO, I. G. Soldagem e Técnicas Conexas: Processos, Laboratório de Soldagem e Técnicas Conexas, UFRGS, Porto Alegre 1996, 477p.

[16] LEWIS, R. W.; MORGAN, K.; THOMAS. H. R.; SEETHARAMU, K. N. The Finite Element Method in Heat Transfer Analysis. $1^{\mathrm{a}}$ ed. Chichester: John Wiley \& Sons Ltd., 1996, 279 p.

[17] BEJAN, A. Transferência de Calor. $1^{\mathrm{a}}$ ed. São Paulo: E. Blücher, 1996, $540 \mathrm{p}$.

[18] AURES, J. E. V. Estudo da Formação, Geometria e Resistência do Ponto na Soldagem por Resistência: Uma Abordagem Estatística. 2006. 164 p. Dissertação de Mestrado em Sistemas Mecatrônicos, Faculdade de Tecnologia, Universidade de Brasília, Brasília.

[19] INCROPERA, F. P.; DEWITT, D. P. Fundamentos da Transferência de Calor e de Massa. $5^{\mathrm{a}}$ ed. Rio de Janeiro: LTC, 2003, 698 p.

[20] Matweb Material Property Data. Disponível em: < $\underline{\mathrm{http}: / /}$ www.matweb.com/search/DataSheet.aspx?12674 >. Acesso em 15 de Maio de 2008.

[21] MatWeb Material Property Data. Disponível em:< http:// www.matweb.com/search/DataSheet.aspx?11859 >. Acesso em 15 de Maio de 2008.

[22] VURAL, M.; AKKUS, A.; ERYÜREK, B. Effect of Welding Nugget Diameter on the Fatigue Strength of the Resistance Spot Welded Joints of Different Steel Sheets, Journal Of Materials Processing Technology, v.176, n 1-3, p.127-132, June 2006.

[23] MARASHI, P.; POURANVARI, M.; SANAEE, S. M. H.; ABEDI, A.; ABOOTALEBI, S. H.; GOODARZI, M. Relationship Between Failure Behavior and Weld Fusion Zone Attributes of Austenitic Stainless Steel Resistance Spot Welds, Materials Science and Technology, v. 24, no 12,p. 1506-1512, December 2008.

[24] GOULD, J. E. An Examination of Nugget Development during Spot Welding, Using Both experimental and Analytical Techniques, Welding Journal, USA, v.66, n.1, p.1s-10s, January 1987.

[25] MatWeb Material Property Data. Disponível em:< http:// www.matweb.com>. Acesso em 4 de Janeiro de 2010. 